\title{
Erratum
}

\section{Erratum to: Fluctuations of the Nodal Length of Random Spherical Harmonics}

\section{Igor Wigman}

Cardiff School of Mathematics, Cardiff University, Senghennydd Road, Cardiff CF24 4AG, UK. E-mail: wigmani@ cardiff.ac.uk

Received: 18 August 2011 / Accepted: 5 September 2011

Published online: 10 November 2011 - (C) Springer-Verlag 2011

Commun. Math. Phys. 298, 787-831 (2010)

There was a sign mistake made in the computation of one of the summands in the expression $\tilde{b}_{n}(\phi)$ defined in (39). Namely, the correct definition is

$\tilde{b}=\tilde{b}_{n}(\phi)=P_{n}^{\prime}(\cos \phi) \cos \phi-P_{n}^{\prime \prime}(\cos \phi)(\sin \phi)^{2}-\frac{P_{n}(\cos \phi)}{1-P_{n}(\cos \phi)^{2}} \cdot P_{n}^{\prime}(\cos \phi)^{2}(\sin \phi)^{2}$, and not as given. Though the main essence stays unimpaired, the leading constant in the main quantities in this paper were effected as a result of the mistake made; in particular the leading constant for the nodal length variance is consistent with Berry's prediction (taking into account the symmetry of the spherical nodal lines), unlike what is claimed in the original version of the manuscript.

That is, the original claim of Theorem 1.1,

$$
\operatorname{Var}\left(\mathcal{Z}\left(f_{n}\right)\right)=\frac{65}{32} \log n+O(1)
$$

should be replaced by

$$
\operatorname{Var}\left(\mathcal{Z}\left(f_{n}\right)\right)=\frac{1}{32} \log n+O(1)
$$

(The references are all with respect to the publication in Communications in Mathematical Physics.) In addition, the definition on p. 793, Eq. (13) of the leading constant $c(\varphi)$, appearing in Theorems 1.4 and 1.5 should be

$$
c(\varphi):=\frac{\|\varphi\|_{L^{2}\left(\mathcal{S}^{2}\right)}^{2}}{128 \pi}>0,
$$


and the definition on p. 794, Eq. (15) of the leading constant appearing in Corollary 1.6 should be

$$
c=c(F):=\frac{|F|}{128 \pi}>0 .
$$

A full list of corrections is available via arxiv, at http://arXiv.org/abs/0907.1648v3 [math.PR], 2011.

Acknowledgement. The author wishes to thank Sven Gnutzmann and Iosif Polterovich for useful conversations which helped him find the mistake in the original manuscript.

Communicated by S. Zelditch 